\title{
Robust Modified MVDR Scheme Using Chirp Signal for Direction of Arrival Estimation
}

\author{
Kalfika Yani, Koredianto Usman and Fiky Y. Suratman \\ ${ }^{1}$ School of Electrical Engineering, Telkom University, Bandung, 40287, Indonesia \\ Corresponding: kalfika.yani@gmail.com
}

Manuscript received Marc 05, 2020; revised June 13, 2020, Accepted June 20, 2020

\begin{abstract}
This research is about an effort to increase the robustness of the Minimum Variance Distortionless Response (MVDR) algorithm to noise by using a chirp signal for direction of arrival estimation (DoA). DoA is a part of radar capability to estimate the angle of arrival on the object under observation. The conventional MVDR as proposed by J. Capon, was designed to work with the monochromatic sinusoidal signal. Even though the conventional MVDR work on low SNR up to $0 \mathrm{~dB}$, however, the conventional method does not work well if chirp signal is used instead of monochromatic sinusoidal signal. The usage of MVDR chirp signal is essential in the case of a very low SNR environment such as in long distance object detection, which is typically more than $10 \mathrm{~km}$. The problem to be solved in this research is how to modify the MVDR algorithm so that it can work well on chirp signal. In this research we offer a modified MVDR algorithm by adding the matched filter and the phase detector components before the MVDR algorithm is applied. Matched filter is responsible for the timing of the chirp signal detection, and the phase detector is to estimate the time delay estimation of each chirp signal from each antenna with a reference signal, which correspond to the phases. Based on the phase estimation, sinusoidal signal is generated and fed to the MVDR algorithm. On the technical aspect, the chirp signal is sent intermittently with a duration of $100 \mu \mathrm{s}$ and repeated in time interval of $1 \mathrm{~ms}$. The antenna sensor using an array of Uniform Linear Array (ULA) which consist of $N$-elements. Computer simulation shows that the modified MVDR using the chirp signal improve the robustness of the algorithm up to $-30 \mathrm{~dB}$, while on the other hand the classical MVDR works only up to $0 \mathrm{~dB} S \mathrm{SNR}$. $-30 \mathrm{~dB}$ of $\mathrm{SNR}$ is the minimum requirement of 3D Radar existing.
\end{abstract}

Keywords: Chirp; Classic MVDR; Modified MVDR; Matched Filter; Phase Detector; DoA

doi: 10.25124/jmecs.v6i1.2630

\section{Introduction}

In 3D radar systems, antenna arrays are used to process beamforming to define the elevation degree, which aims to determine the height of target. On the conventional transmitter radar, the cosecant beam pattern is produced by parabolic antenna. In 3D radar, the antenna is an arrays antenna, so to get cosecant beam we must use phase shifter. To determine the height of a target, the receiver uses Direction of Arrival (DoA) technique. DoA is processed in software. With the DoA process, the target height can be estimated. There are three popular of classical DoA algorithms, which are: Minimum Variance Distortionless Response (MVDR), Multiple Signal Classification (MUSIC), and Estimation of Signal Parameters Via Rotational Invariant Techniques (ESPRIT). In 3D radar pulse compression, the azimuth is calculated from range resolution of pulse compression [1].
MVDR algorithm was proposed by Capon in 1969 [2]. The algorithm utilized the property of the orthogonality between the inverse of covariance matrix of received signals to the actual direction of arrival, as represented by antenna steering vector [3]. The main advantage of MVDR algorithm is its robustness against noise. In addition, MVDR has low complexity. While the drawback of this algorithm is that it has a low detection resolution. Low detection resolution means that it usually cannot distinguished two objects with a small angle of arrival difference. In the term of implementation, MVDR is favourable to other algorithm such as MUSIC and ESPRIT. The application of MVDR in real life situation are, for examples, continuous wave $(\mathrm{CW})$ radar system using MVDR to detect the elevation angle [4], shallow water sonar processing [5], and through the wall radar imaging [6].

MUSIC Algorithm was proposed by Ralph O. Schmidt [7]. MUSIC algorithm is one of the breakthrough algorithms in the field of DoA with 
eigen analysis techniques on covariance matrix. This algorithm is also called as an eigen based algorithm. The success of this algorithm in detecting several sources at once with very high resolution become the main attraction of this algorithm. Given this advantage, MUSIC algorithm is sensitive to the noise, also number of samples for calculating the covariance matrix must be large enough to obtain a statistical stability. In addition, MUSIC also has a high computation complexity [8].

ESPRIT algorithm was proposed by Roy, Paulraj and Kailath [9]. ESPRIT algorithm exploits the symmetry structure of the ULA arrangement with the exploitation of this symmetry structure, then the ESPRIT algorithm doesn't do corner-by-corner scanning. However, analytically obtains DoA estimates from received signal and exploited this signal from antenna symmetry structure of array antennas. Using this symmetric structure, ESPRIT algorithm does not need to compute the corner to corner scanning, thus it offers faster computer speed. The weakness of the ESPRIT algorithm is ability the detection is only half the signal compared to the algorithm MUSIC. The computational process in ESPRIT is quite heavy and requires high computing resources. This reason leads to ESPRIT is less popular in the field of implementation [9]. Because of this complexity, many researchers try to modify and simplify the algorithm. For example, M. Hardt simplified the complex valued computation using unitary transformation, so that the complexity is reduced. His modification is called unitary ESPRIT algorithm [10].

Even though MVDR is popular for implementation, MVDR cannot address a more practical issue such as low noise environment in the case of military radar. Due to requirements of last working distance (up to $10 \mathrm{~km}$ ). it is a common situation that the received signal is less than $0 \mathrm{~dB}$. In this case, conventional MVDR is usually failed to give a satisfactory result. In this research we proposed the modified of MVDR algorithm using chirp signal, with adding matched filter and phase detector before MVDR processing. Using this modified system, we expect to solve the problem of low SNR condition.

\section{System Modelling}

\subsection{Antenna Design}

The antenna used as a basic structure for DoA estimation is an array antenna arranged in a Uniform Linear Array (ULA), and assumed to be an isotropic antenna. The advantage of ULA is simple and uniform. With this uniform and simple structure, the estimation of DoA can be reduced to a simple mathematical equation.

Antennas are arranged vertically against the horizon plane with $\mathrm{M}$ elements, the distance between elements is constant value of $d$, and the signal source comes from a relatively far distance with the angle of arrival $\theta$, the beam reaching for each antenna is describe in Figure 1.

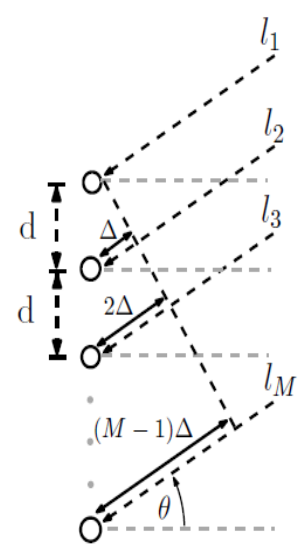

Figure 1: M-Array Antenna

By using the first antenna as a reference, the direction of the incoming signal will cause a difference in the distance of each element of the adjacent antenna by $\Delta$ which is expressed as the following equation:

$$
\Delta=d \sin (\theta)
$$

$d$ is the distance between the antennas and the angle of arrival $\theta$. Assuming the received signal is a sinusoidal signal, the wavelength is $\lambda$, and the phase difference between the two antenna elements $\delta$ :

$$
\delta=\frac{2 \pi}{\lambda} d \sin (\theta)
$$

The system applied in pulse compression 3D Radar system with technical requirements is given in Table 1.

Table 1: RF Requirements

\begin{tabular}{|l|l|}
\hline RF Frequency & $3 \mathrm{GHz}$ \\
\hline M-Array Antenna & 4,8 and 16 \\
\hline Beam width & $0^{\circ}-70^{\circ}$ \\
\hline
\end{tabular}

\subsection{Classic MVDR System Modelling}

In the classic MVDR, the chirp signal is directly 
process in MVDR. This condition is given in Figure 2. In the figure, we observe that the classical MVDR algorithm works on monochromatic sinusoidal. The angle of arrival of the source will directly correspond to the phase delay of adjacent antennas as given in Equation (2).

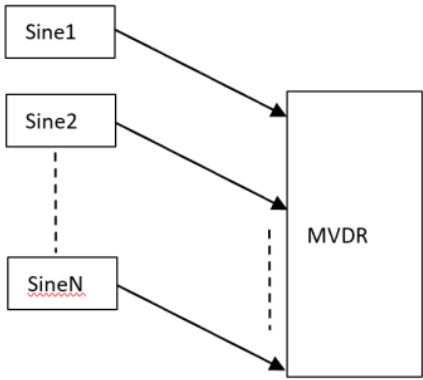

Figure 2: Classical MVDR

\subsection{Modified MVDR System Modelling}

The input used in the conventional MVDR algorithm is the sine signal with frequency and singlephase values. In this thesis the pulse compression LFM (chirp) signal will be used as the input. The proposed scheme is given in Figure 3.

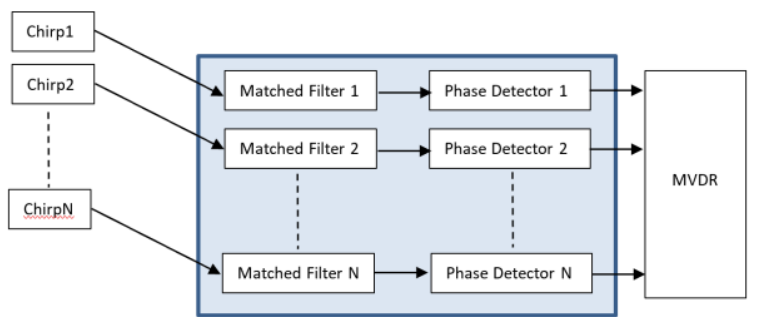

Figure 3: Modified MVDR System Design

The input signal is Non Linear FM chirp signal with parameters in Table2.

Table 2: Input Signal Requirements

\begin{tabular}{|l|l|}
\hline Sampling Frequency & $4 \mathrm{MHz}$ \\
\hline Bandwidth Signal & $2 \mathrm{MHz}$ \\
\hline Pulse Repetition Interval & $1 \mathrm{~ms}$ \\
\hline Duty Cycle & $10 \%$ \\
\hline Chirp Phase & Non Linear FM \\
\hline
\end{tabular}

The input signal (chirp) will transmit in $(\tau) 100$ $\mu \mathrm{s}(10 \%$ of PRI), with bandwidth B is $2 \mathrm{MHz}$, which can be represented as (3).

$$
\operatorname{chirp}=e^{\left(j\left(\pi\left(\frac{B}{\tau}\right) *\left(t-\frac{\tau}{2}\right)\right)\right)^{2}}
$$

The chirp signal transmits in every $1 \mathrm{~ms}$, as describe in Figure 4.
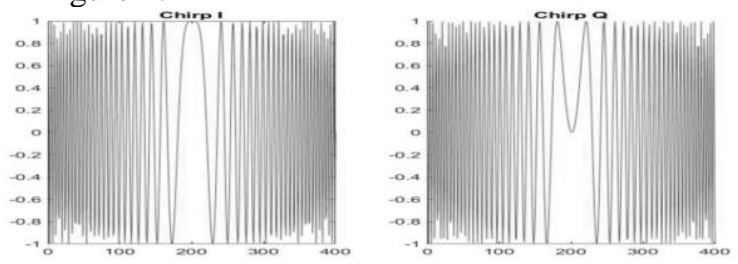

Figure 4: Chirp Transmit Signal

The chirp signal has different phase when transmit, and the monochromatic sinusoidal have the same phase in t time, as show in Figure 5.

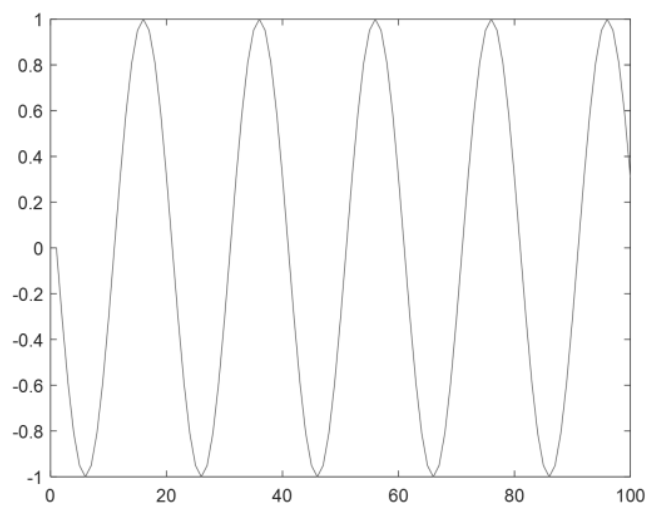

Figure 5: Monochromatic Sinusoidal

The receiver signal in first antenna and the others is delay in N-samples. The input chirp with 4 ULA is given in Figure 6.
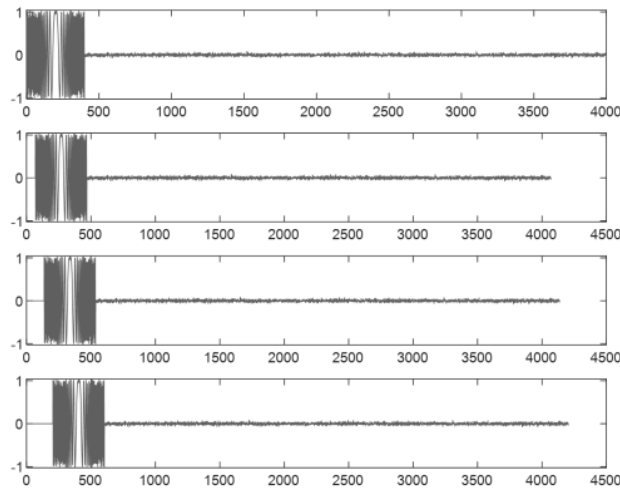

Figure 6: Chirp in Receiver

In order to detect chirp signals, matched filters are used on the receiver. The matched filter coefficient is derived from the value of chirp real and imaginer, which are called in Numerator on fdatool. Matched filter processes the correlation between the chirp signal that comes with the filter coefficient. If what is received is matched, it will produce a high peak. The matched filter is operated in 0 to $\tau$ second, so the result 
just in that period, and it is zeros result in another time, as in (3).

$$
x(t)= \begin{cases}1, & 0 \leq t \leq \tau \\ 0, & \text { otherwise }\end{cases}
$$

The output of matched filter for 4 ULA is given in Figure 7.

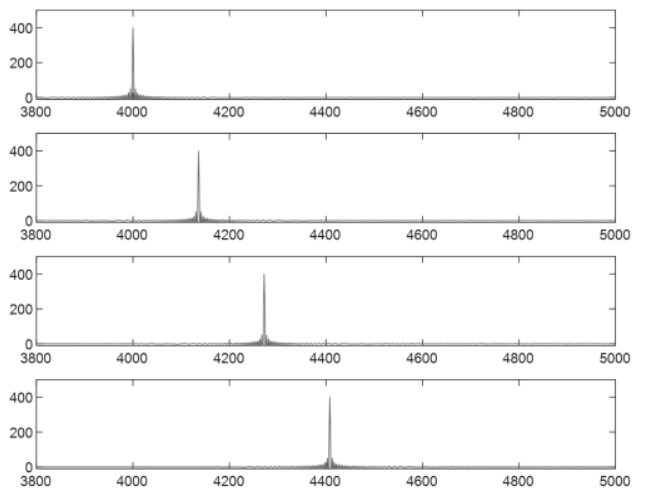

Figure 7: Output Matched Filter

The phase detector method in this research is to convert from the time delay signal into phase. Input signal from matched filter processing will compare with phase reference. Output of phase detector is the angle from each array antennas in ULA. The phase detector can define in Figure 8 .

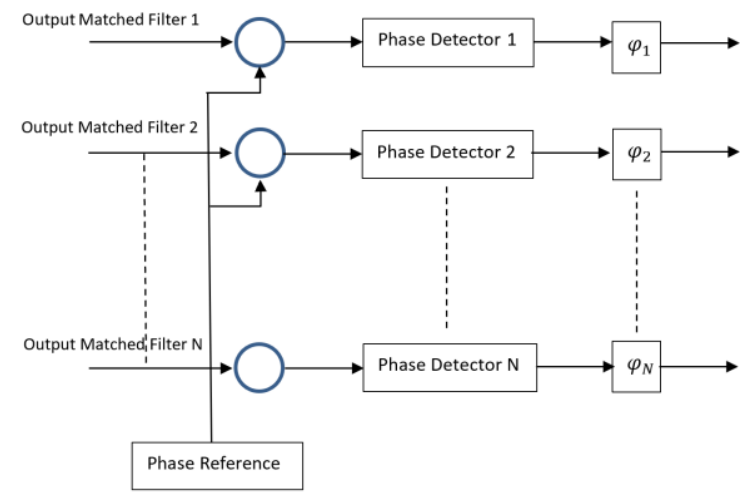

Figure 8: Phase Detector

The phase reference comes from the matched filter output 1. Each matched filter output 1, 2 until N will compared with the phase reference. Difference in value from each output matched filter with phase reference, will produce a certain phase value that will be issued by the phase detector. The output of the phase detector will be forwarded to the MVDR for further calculation of the DoA value.

When the angle of arrival signal is come from $90^{\circ}$, the maximum distance between two antennas is $\mathrm{d}$ samples, the difference between two antennas is $\mathrm{n}$ sample, so the angle of arrival can be defined as (4).

$$
\varphi=\sin ^{-1}(n / d)
$$

In the implementation of phase detector FPGA, angle $\varphi$ implemented using lookup table to save time calculation.

In MVDR algorithm process, first step is determining the number of sources, angle of arrival, number of antennas and antenna spacing in accordance with predetermined conditions. Next step is to generate the signal chirp with the specified signal specifications. After that is to generate the steering vector to direct the signal to a certain angle. After generating the steering vector emulate both the chirp signal and the steering vector to get the received signal. Signal that has been emulated, enter the noise that will be used to calculate the SNR. In order to improve the stability of signal estimation in noisy environments, this algorithm collects considerable data and processes it into a covariance matrix $\left(R_{x x}\right)$. In last step the MVDR power spectrum $\left(P_{M V D R}\right)$ is calculated by (5).

$$
P_{M V D R}(\theta)=\frac{1}{a^{T}(\theta) R_{x x}^{-1} a(\theta)}
$$

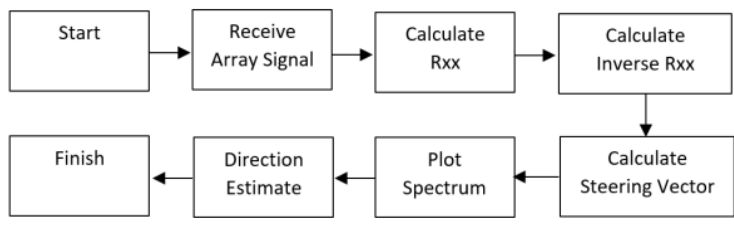

Figure 9: MVDR Algorithm Flowchart

\section{Result and Analysis}

In this section, we perform accuracy of modified MVDR computer simulation to access the performance of Modified MVDR algorithm. As reference we use classical MVDR as comparison method. The simulation is conduced of measure of the accuracy, resolution, and computation time.

\section{1 The Accuracy as function of SNR}

Accuracy is the ability of the algorithm to estimate the angle of arrival. In this research, the level of accuracy is determined by comparing the angle of transmit signal (beam steering) with the angle of arrival estimation on classical MVDR and modified MVDR. The beam steering is $30^{\circ}$. 
In first simulation using $\mathrm{SNR}-30 \mathrm{~dB}$, and $30^{\circ}$ transmit beam steering. As the result 4 ULA classical MVDR have accuracy $4.3245^{\circ}$ of MSE, while 4 ULA modified MVDR have better accuracy $0.5^{\circ}$ of MSE, as in figure 10.

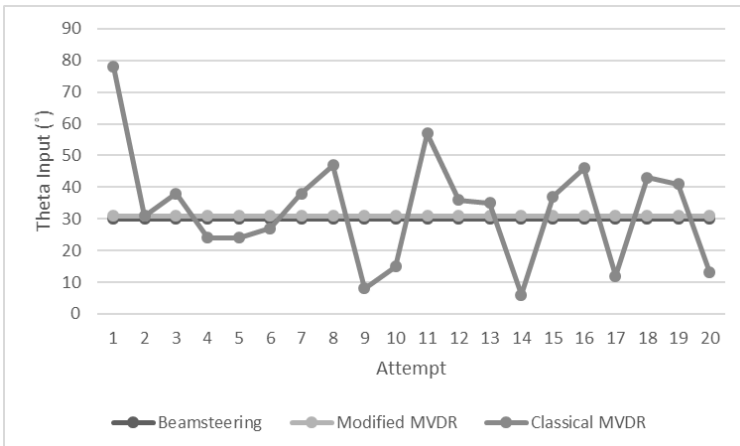

Figure 10: Accuracy table for $4 \mathrm{ULA}, \mathrm{SNR}=-30 \mathrm{~dB}$

In second simulation, SNR $-30 \mathrm{~dB}$, and $30^{\circ}$ transmit beam steering, 8 ULA used. As the result classical MVDR have $4.802^{\circ}$ of MSE, while modified MVDR have $0.5^{\circ}$ of MSE, as in figure 11 .

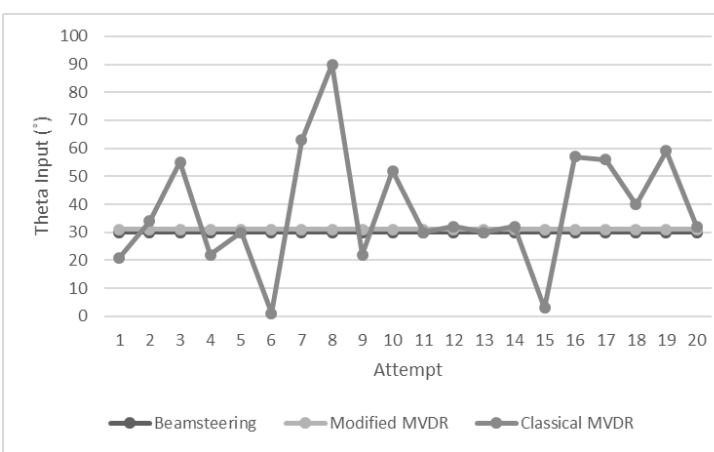

Figure 11: Accuracy table for $8 \mathrm{ULA}, \mathrm{SNR}=-30 \mathrm{~dB}$

In last simulation SNR $-30 \mathrm{~dB}$, and $30^{\circ}$ transmit beam steering, 16 ULA used. As the result classical MVDR have $1.28125^{\circ}$ of MSE and modified MVDR have $0.5^{\circ}$ of MSE, as in figure 12 .

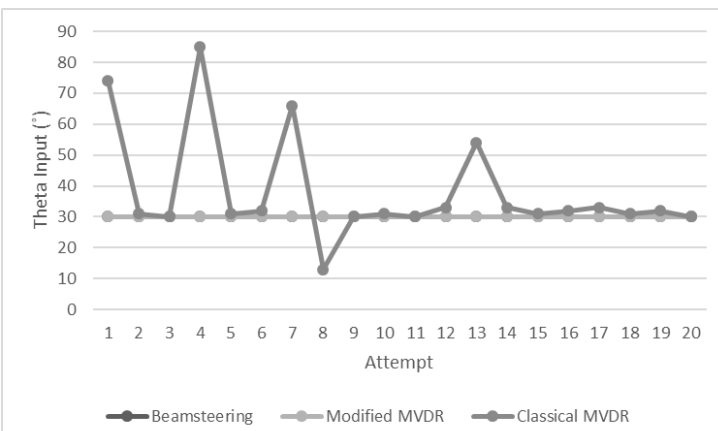

Figure 12: Accuracy table for 16 ULA, SNR $=-30$ $\mathrm{dB}$
Based on Figure 10, Figure 11, and Figure 12, Modified MVDR Algorithm is more robust than Classical MVDR, because Modified MVDR can work properly in SNR $=-30 \mathrm{~dB}$, while Classical MVDR work properly in $0 \mathrm{~dB}$ minimum of SNR.

\subsection{Resolution}

Resolution is the ability of an algorithm to estimate the angle of arrival from two target from different angles in the same time. In this simulation, the 2 target comes between angle $0^{\circ}$ to $70^{\circ}$ in several times.

From simulation show that classic MVDR with 0 SNR in 4 ULA and 8 ULA fail to detect the DoA. On the other hand, if classic MVDR using 16 ULA, target detected with $20^{\circ}$ of resolution. With maximum angle can reach is $70^{\circ}$. In Figure 13.a show that the classic MVDR can detect $50^{\circ}$ and $70^{\circ}$.

From the simulation of Modified MVDR with SNR $-30 \mathrm{~dB}, 16$ ULA show that target can be detected with $1^{\circ}$ resolution.

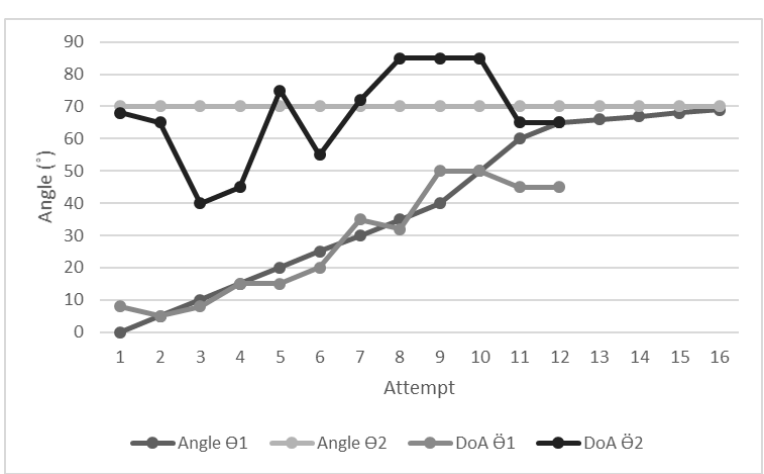

Figure 133.a: Resolution table of classical MVDF for $16 \mathrm{ULA}, \mathrm{SNR}=-30 \mathrm{~dB}$

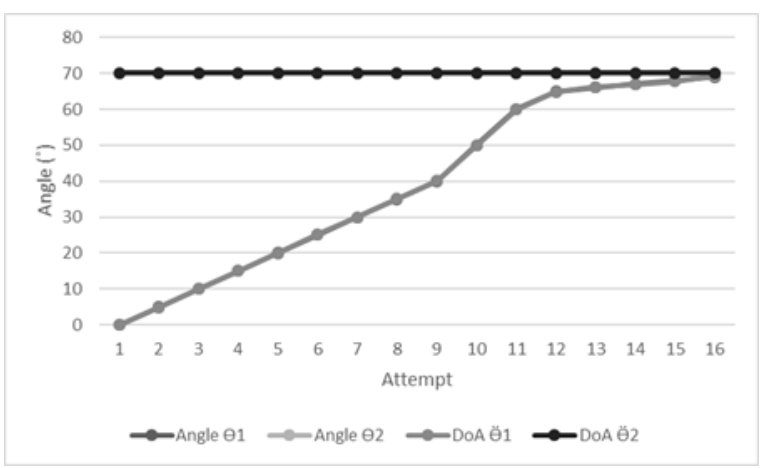

Figure 13.b: Resolution table of modified MVDF for $16 \mathrm{ULA}, \mathrm{SNR}=-30 \mathrm{~dB}$ 
Based on Figure 13.a and Figure 13.b, Modified MVDR is more robust than Classical MVDR. In SNR $-30 \mathrm{~dB}$, Modified MVDR have $1^{\circ}$ resolution, while Classical MVDR have $20^{\circ}$ resolution.

\subsection{Simulation Time}

The modelling and simulation are using computer with Intel Core i7 $8^{\text {th }}$ generation, with 16 GB of RAM. Simulation is done with two scenarios. In the first scenario, input is continuous sinus signal which process directly using classical MVDR algorithm. This simulation takes 0.0679 second. In second scenario, the input is chirp signal and process using modified MVDR, which consist of matched filter and phase detector in pre-processing, and then processed by MVDR algorithm. This simulation takes 0.2001 second in averages.

\section{Conclusion}

The system requirement in this research is based on pulse compression 3D Radar surveillance systems. If the arrival signal come from one source, phase detector can estimate the angle of arrival properly, but if the receive source more than one, we need MVDR to estimate their angle of arrival.

Classic MVDR can work properly in minimum SNR $0 \mathrm{~dB}$, and the array of antennas minimum in 16 ULA. But, the 16 ULA of classic MVDR only have 20 degree of resolution in $0 \mathrm{~dB}$ of SNR.

In the other hand, using modified MVDR, the algorithm can estimate the angle of arrival with 1 degree of deviation using 4 ULA, 8 ULA and 16 ULA in SNR up to $-30 \mathrm{~dB}$. It means the modified MVDR is very robust with matched filter in pre-processing. With the resolution of modified MVDR is 1 degree, the algorithm can detect the 2-arrival object with 1degree angle of arrival with up to $-30 \mathrm{~dB}$ of SNR. As conclusion the modified MVDR can implement for DoA in 3D Radar surveillance systems.

The input chirp signal is NLFM, with duty cycle $10 \%$. For next research to increase the performance of modified MVDR, the input signal should be modified with LFM and reduce the duty cycle.

\section{References}

[1] M. D. Anderson, "Resolution in Radar Mapping," Naval Postgraduate School, no. Monterey; California, March 1993.

[2] J. Capon, "High-Resolution FrequencyWavenumber Spectrum Analysis," IEEE Military Communications (MILCOM), vol. 3, no. Oct. 1986, 1986.
[3] K. Usman, Estimasi Arah Kedatangan SInyal dengan Penginderaan Komprehensif, Bandung: ITB, 2019.

[4] R. M. Hedge, Y. Jin and B. D. Rao, "Spectral Estimation Of Voiced Speech Using A Family of MVDR Estimates," ICASSP, no. 2007, 2007.

[5] A. A. Blomberg, A. Austeng and R. E. H. S. A. V. Synnes, "Improving Sonar Performance in Shallow Water Using Adaptive Beamforming," IEEE Journal Oceanic ENgineering, vol. 38, no. April 2013, p. 2013.

[6] Yoon, Y.-S. \&. Amin, M. \&. Ahmad and Fauzia, "MVDR Beamforming for Throughthe-Wall Radar Imaging, Aerospace and Electronic Systems," IEEE Transaction, vol. 47, no. 2011, pp. 347-366, 2011.

[7] R. O. Schmidt, "Multiple Emitter Location and Signal Parameter Estimation," IEEE Transactions on Antennas and Propagation, Vols. ap-34, no. September 1976, 1986.

[8] D. Spielman, A. Paulraj and T. Kailath, "Performance Analysis of The MUSIC Algorithm," IEEE International Conference on Accoustic, Speech, and Signal Processing, no. Tokyo, Japan, pp. 1909-1912, 1986.

[9] R. Roy and T. Kailath, "ESPRIT-Estimation of Signal Parameters via Rotational Invariance Techniques," Proceeding of IEEE Military Communications (MILCOM) ConferenceCommunications, vol. 7, no. 1989, 1989.

[10] M. Haardt and J. Nossek, "Unitary ESPRIT: How to Obtain Increased Estimation Accuracy with A Reduced Computational Burden," IEEE Transactions on SIgnal Processing, vol. 43, no. May 1995, p. 5, 1995.

[11] S. P. Applebaum, "Adaptive Array," IEEE Transactions on Antennas and Propagation, Vols. Ap-24, no. 1986, p. 5, 1986.

[12] B. Mahafza, Radar Signal Analysis and Processing Using Matlab, A Chapman \& Hall Book, 2009.

[13] M. Zoltowski, "On the Performance Analysis of the MVDR Beamformer in the Presence of Correlated Interference," IEEE Transactions on Accoustics, Speech, and Signal Processing, 1998.

[14] M. Z. a. C. Mathews, "DOrection FInding with Uniform Circular ARray via Phase Mode Excitation and Beamspace Root-MUSIC," IEEE Proceeding, 1992.

[15] S. Wenjing, D. Zheng and X. W. a. L. Yingsong, "Digital Beamforming Based on FPGA for Phased Array Radar," Progress in Electromagnetics Research Symposium, no. St. Petersburg, Russia, 2017. 
[16] W. Christian, "Antenna Techniques," radartutorial.eu, 2016.

[17] E. M. A. Mahdi, "Distributed Arithmetic Analysis, Design and Applications," Computer ENgineering Department Libyan Academy Misurata, no. 5 September 2015, 2015.

[18] M. Labitt, "Obtaining Low Sidelobes Using Non-Linear FM Pulse Compression," Lincoln Laboratory, Massachusetts Institute of Technology, Lexington, Massachusetts, 1994.

[19] M. R. Widyantara, Sugihartono, F. Y. Suratman, S. Widodo and P. Daud, "Analysis of Non Linear Frequency Modulation (NLFM) Waveforms for Pulse Compression Radar," Jurnal Elektronika dan Telekomunikasi (JET), vol. 1, no. 2018, pp. 27-34, 2018.

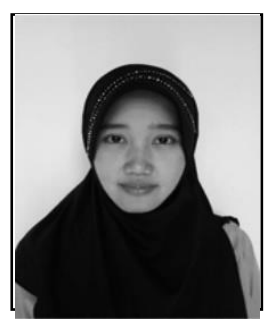

Kalfika Yani S.T., IPM., ASEAN Eng., was born on 15 September 1985. She was studied Bachelor Degree in 2008 from STT Telkom Bandung, Indonesia majoring in Telecommunication Engineering. During 2008-2020 she works in PT. Len Industri (Persero) as a Digital FPGA Engineer, and developed signal processing for Manpack Radio and Radar Signal Processing for 2D SBand Surveillance Radar and 3D Air Surveillance Radar.

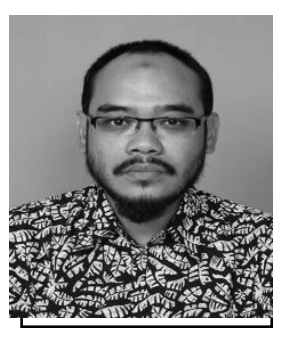

Dr. Ing Fiky Y. Suratman, S.T., M.T. was born in Jakarta, Indonesia, in 1976. He received Bachelor Degree from Engineering Physics and Master Degree from School of Electrical and Informatics (STEI), Institut Teknologi Bandung, Bandung, Indonesia in 1998 and 2006, respectively. He was awarded scholarship from German Academic Exchange Service (DAAD) to continue his study at Technische Universitaet Darmstadt (TUDarmstadt), Germany, until he recived his Dr.-Ing. Degree $(\mathrm{PhD})$ in 2014. Early placement in industry (Astra Microtronics Technology) from 1998 to 2001, was then followed by lectureship in Universitas Komputer Indonesia (UNIKOM). In 2007, he joined the Faculty of Electrial Engineering, Institut Teknologi Telkom (now Universitas Telkom) as a faculty member. $\mathrm{He}$ is the head of Master Degree of Electrical Engineering, Telkom University since 2014. He is a member of IEEE (Signal Processing Soiciety and Communications Society), and since 2016 he is the chair of Signal Processing Society (SPS) Indonesia Chapter. His research interest lies on Statistical Signal Processing and Radar Signal Processing.

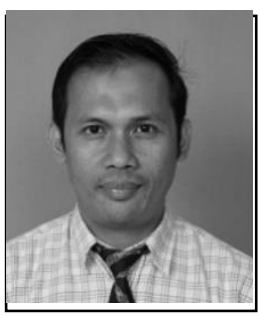

Dr. Koredianto Usman, S.T., M.Sc. was born in Sumatera Selatan Indonesia October 2, 1975. His obtained his bachelor from Electrical Engineering Bandung Institute of Technology, Indonesia, 1999, and master degree from Munich Institute of Technology, Germany in 2001. In 2019, he obtained a PhD in Electrical and Informatics, Engineering from Bandung Technology Institute. His research interest is signal processing, and he has been publishing journal articles in various international proceedings and national journals. Besides, he is an active reviewer at international telecommunication conferences. 